\title{
Avaliação de um esquema de realimentação utilizado após 43 dias de jejum voluntário
}

\author{
Evaluation of a refeeding plan employed \\ after 43 days of voluntary fasting
}

Nídia Denise PUCCl

Belchor FONTES 2,3

Renato Sérgio POGGETTI2,3

RE S U M O

\section{Objetivo}

Avaliar um esquema de realimentação utilizado em pacientes após jejum prolongado voluntário.

\section{Métodos}

Foi realizado estudo retrospectivo, com base em levantamento dos prontuários de 7 pacientes (média de idade de 41,6 anos e desvio-padrão=5,3) em jejum voluntário por 43 dias em jejum seguidos de 14 dias de realimentação. As variáveis estudadas foram: antropométricas, clínicas, dietéticas, laboratoriais e avaliação da composição corpórea por bioimpedância. Foram utilizadas as seguintes dietas por via oral na realimentação: oligomérica enriquecida com glutamina, associada à dieta hipogordurosa e isenta de lactose (primeira semana); dieta branda normolipídica com lactose (segunda semana). A análise descritiva dos dados apresentados incluiu média e desvio-padrão. A análise de variância ou o teste não paramétrico de Kruskal-Wallis foram utilizados para comparação dos dados nos períodos de jejum e realimentação. Utilizou-se o programa estatístico SIGMA STAT versão 2,03 sendo considerado o nível de significância de 5\%.

\section{Resultados}

No jejum houve redução significativa do peso corpóreo $(18,0 \%$ desvio-padrão=2,3), da prega cutânea do tríceps $(48,2 \%$ desvio-padrão=9,0) e da hemoglobina $(15,98 \mathrm{~g} / \mathrm{dL}$ desvio-padrão=0,99 para 12,74g/dL desvio-padrão=0,47). Na segunda semana de realimentação houve aumento significativo na contagem total de linfócitos (954 mil/ $\mathrm{mm}^{3}$ desvio-padrão=242 para $1619 \mathrm{mil} / \mathrm{mm}^{3}$ desvio-padrão=232) e dos distúrbios gastrointestinais, em relação à primeira semana. Não ocorreu Síndrome da Realimentação.

${ }^{1}$ Universidade de São Paulo, Faculdade de Medicina, Hospital das Clínicas, Divisão de Nutrição e Dietética. São Paulo, SP, Brasil.

2 Universidade de São Paulo, Faculdade de Medicina, Hospital das Clínicas, Departamento de Cirurgia, Serviço de Cirurgia de Emergência e Divisão de Clínica Cirúrgica III. São Paulo, SP, Brasil.

${ }^{3}$ Universidade de São Paulo, Hospital das Clínicas, Faculdade de Medicina. Av. Dr. Enéas Carvalho Aguiar, 255, Cerqueira César, 05503-000, São Paulo, SP, Brasil. Correspondência para/Correspondence to: N.D. PUCCI. E-mail: <nidiapucci@ig.com.br>. 
504 | N.D. PUCCl et al.

\section{Conclusão}

O esquema de realimentação utilizado promoveu resultado global adequado, mas requer redução do aporte energético e definição de momento mais adequado para reintrodução de lactose e da dieta normolipídica.

Termos de indexação: Jejum prolongado voluntário. Métodos de realimentação. Avaliação nutricional.

\section{A B S T R A C T}

\section{Objective}

To evaluate a refeeding plan employed in patients after a long period of voluntary fasting.

\section{Methods}

A retrospective study was done by reviewing the medical records of 7 male patients (mean age of 42.0 years; standard deviation of 5.3 years) who were refed for 14 days after a 43-day fast. The studied variables were: clinical, anthropometric; biochemical and body composition by bioimpedance analysis. The following diets were used orally in the refeeding program: glutamine-enriched oligomeric diet associated with a low-fat lactose-free diet (first week); normal-fat diet with lactose (second week). Descriptive analysis of the data included mean and standard deviation. Analysis of variance or the non-parametric Kruskal-Wallis test was used to compare data during the fasting and refeeding periods. The SIGMA STAT statistics software package version 2.03 was used to analyze data with the significance level set at $5 \%(p<0.05)$.

\section{Results}

During the fasting period there was a significant reduction in body weight (18.0\%; standard deviation=2.3), triceps skinfold (48.2\%; standard deviation=9.0) and hemoglobin concentration (from 15.98g/dL; standard deviation=0.99 to $12.74 \mathrm{~g} / \mathrm{dL}$; standard deviation=0.47). During the second week of refeeding, there was a significant increase in total lymphocyte count (from 954 thousand/mm3; standard deviation=242 to 1.619 thousand//mm3; standard deviation=232); and gastrointestinal problems when compared with the first week. No case of refeeding syndrome was observed.

\section{Conclusion}

The refeeding program used promoted a satisfactory global result but it is necessary to reduce energy intake and define the best moment to reintroduce lactose and a normal-fat diet.

Indexing terms: Prolonged voluntary fasting. Refeeding methods. Nutritional assessment.

\section{N T R O D U ÇÃ O}

O jejum é conceituado como a ausência da ingestão de alimentos e de nutrientes por um período mínimo de 6 horas, sendo considerado prolongado quando superior a 72 horas. O jejum prolongado ocorre com freqüência em pacientes hospitalizados em preparo para exames diagnósticos ou em períodos pré-operatórios, podendo ocasionar alterações metabólicas e nutricionais, além de contribuir para o aumento da prevalência de desnutrição hospitalar ${ }^{1-3}$. Voluntariamente, o jejum prolongado é praticado por motivos religiosos ou por greve de fome ${ }^{4}$. As principais alterações fisiológicas observadas no jejum prolongado incluem uma redução acentuada da gordura corpórea devido à utilização desta para produção de energia, com conseqüente aumento dos ácidos graxos plasmáticos. Incluem também a perda de massa muscular, que ocorre em menor proporção devido à preservação de proteínas necessárias às funções celulares essenciais. Geralmente ocorre também redução do número de neutrófilos, de linfócitos, e dos níveis séricos de glicose, insulina, albumina e, mais tardiamente, da hemoglobina e de vitaminas ${ }^{1,5,6}$.

São manifestações clínicas comuns no jejum prolongado a perda progressiva de peso, a sensação de fraqueza e a presença de anemia, edema e propensão a infecções ${ }^{5-8}$. No trato gastrointestinal, são relatadas redução da produção de enzimas digestivas, atrofia da mucosa intestinal com redução da altura das vilosidades, alteração 
do transporte de aminoácidos através da membrana celular, e redução da absorção de glutamina e arginina, comprometendo a digestão e a absorção de nutrientes na fase de realimentação9. Tais distúrbios podem contribuir para intolerância alimentar, tornando necessário um período de vários dias a semanas para que a atividade das enzimas digestivas e dos aminoácidos seja restabelecida ${ }^{9,10}$.

As complicações metabólicas decorrentes da realimentação após jejum prolongado foram inicialmente relatadas em sobreviventes dos campos de concentração e associadas a inanição grave, e constituem a entidade clínica atualmente denominada síndrome da realimentação $0^{5,11}$. Esta síndrome pode ocorrer tanto na realimentação oral como na enteral ou parenteral. É caracterizada por redução da concentração sérica de eletrólitos (fósforo, magnésio e potássio) e, até mesmo, alterações da homeostase da glicose, principalmente na realimentação com carboidratos ou com aporte energético excessivo. Geralmente ocorre na realimentação após jejum superior a 7 dias, em associação a condições diversas, como deficiências nutricionais prévias, período pré-operatório de cirurgias de grande porte, diabetes mellitus descompensado, quimioterapia, nutrição parenteral sem adequada administração de vitaminas ou minerais, administração de aporte energético excessivo e, ainda, na falta de monitoramento e reposição adequada de eletrólitos ${ }^{12-14}$.

Como os distúrbios da síndrome podem levar à falência multiorgânica, sua prevenção na fase de realimentação após jejum prolongado se justifica e requer adequado monitoramento de eletrólitos e controle das calorias administradas aos pacientes, sobretudo no início da realimentação ${ }^{12-14}$.

O jejum voluntário em indivíduos saudáveis, embora raramente observado na prática clínica, representa uma situação peculiar por permitir identificar, na ausência de comorbidades, as alterações fisiológicas específicas decorrentes do jejum, situação propícia para avaliação dos esquemas de realimentação utilizados. A difi- culdade na determinação de um esquema dietético a ser utilizado na realimentação após jejum prolongado decorre do fato de as recomendações dietéticas existentes serem genéricas, visando evitar a síndrome da realimentação, sendo utilizadas como guias, porém passíveis de aprimoramento. Recomenda-se, por exemplo, que a realimentação seja iniciada com aporte energético de $20 \mathrm{kcal} / \mathrm{kg}$ de peso/dia nos primeiros 3 a 5 dias após o término do jejum, com aumento gradativo até atingir as necessidades do paciente (30kcal/ $\mathrm{kg}$ de peso/dia) por volta do $10^{\circ}$ ao $14^{\circ}$ dia ou conforme a tolerância à dieta ${ }^{12,13}$. Não estão bem definidas as formulações ou composições das dietas, nem os períodos em que devem ser administradas aos pacientes visando obter-se recuperação nutricional adequada, breve e livre de intercorrências.

Nesse contexto, o objetivo do presente estudo foi avaliar, retrospectivamente, as repercussões nutricionais do jejum prolongado e, sobretudo, a adequação de um esquema de realimentação utilizado em um grupo de pacientes, após jejum prolongado voluntário.

\section{MÉ TO D O S}

O projeto do presente estudo foi aprovado pela Comissão de Ética para Análise de Projetos de Pesquisa do Hospital das Clínicas e da Faculdade de Medicina da Universidade de São Paulo (FMUSP). Os prontuários médicos dos pacientes foram revisados retrospectivamente, tendo sido realizada a coleta dos dados referentes aos períodos de jejum e à realimentação de sete pacientes do sexo masculino, adultos, previamente hígidos, que estiveram internados na Unidade de Emergências Médicas do Instituto Central do Hospital das Clínicas da FMUSP, por motivo de greve de fome. A revisão dos prontuários abrangeu os 11 dias imediatamente precedentes à internação, nos quais os pacientes permaneceram em jejum na Casa de Detenção em São Paulo. Nesse período de 11 dias, alguns dados registrados pela equipe de saúde da Casa de Detenção foram 
coletados para o presente estudo, tais como peso diário e sintomas. No $11^{\circ}$ dia do jejum os pacientes foram encaminhados ao HCFMUSP, onde continuaram em jejum por mais 32 dias, sendo a seguir submetidos à realimentação durante 14 dias. No $14^{\circ}$ dia da realimentação, portanto, após 46 dias de internação, os pacientes receberam alta hospitalar e foram encaminhados à Casa de Detenção de onde eram provenientes.

Nos prontuários consta que o jejum iniciouse e foi mantido com a recusa total de alimentos e com aceitação irregular de água pelos pacientes por um período de 11 dias durante os quais receberam cuidados da equipe de profissionais de saúde da Casa de Detenção onde permaneceram detidos. Consta também que, por apresentarem fraqueza, tonturas, sinais de desidratação, e elevada perda de peso, foram encaminhados, no $11^{\circ}$ dia do jejum, ao ICHC FMUSP. Nesta instituição mantiveram-se em jejum, recebendo cuidados da equipe multiprofissional (médicos, nutricionistas, e membros da equipe de enfermagem) que registrou os dados utilizado no presente trabalho.

O esquema dietético empregado constou de formulações já padronizadas pelo hospital onde se encontravam os pacientes ${ }^{15}$, elaboradas pela equipe de nutricionistas e administrada por via oral em duas etapas (semanas). Na primeira semana, foi administrada dieta líquida sem resíduo (chá, gelatina e sopa liquidificada), isenta de lactose, seguida de dieta hipogordurosa sólida com baixo teor de resíduos, associadas à dieta de formulação oligomérica enriquecida com até $34 \mathrm{~g}$ de glutamina/dia. Na segunda semana, foi administrada dieta branda normolipídica, com lactose ${ }^{15}$, adaptada individualmente com base na aceitação, tolerância e hábito intestinal dos pacientes. Os registros de aceitação alimentar foram diários, e permitiram o cálculo da ingestão energético-protéica individual realizado para este estudo.

Em síntese, foram coletados dos prontuários dados antropométricos, laboratoriais, clínicos e dietéticos registrados pela equipe multiprofissional do hospital e resultados da bioimpedância: peso diário, índice de massa corpórea (IMC) calculado semanalmente pela equação: IMC=[(peso em kg) / (altura em metro $\left.)^{2}\right]$, medidas da prega cutânea tricipital (PCT) e circunferência do braço (CB), realizadas por meio de técnicas usuais ${ }^{16-18}$, dados laboratoriais: glicemia, hemograma completo, proteínas totais e frações, eletrólitos e minerais; dados referentes ao estado geral, nível de consciência, hábito intestinal diário (número de evacuações e consistência das fezes), manifestações clínicas gerais e gastrointestinais; registros diários das dietas prescritas, oferecidas e aceitação pelos pacientes, bem como as manifestações clínicas decorrentes da introdução das mesmas na realimentação.

No $32^{\circ}$ dia do jejum e no $8^{\circ}$ dia da realimentação constam registros da avaliação da composição corpórea determinada por bioimpedância (aparelho Byodinamics 310 - RJL, Comp Corp Byodinamics) ${ }^{18,19}$, os quais foram coletados do prontuário: massa magra, gordura, água corpórea total, e valores de resistência e da reactância.

A análise descritiva dos dados apresentados incluiu média e desvio-padrão. A análise de variância (ANOVA) ou teste não paramétrico de Kruskal-Wallis foi utilizado para comparação dos dados nos períodos de jejum e realimentação. Utilizou-se o programa estatístico SIGMA STAT versão 2,03 sendo considerado nível de significância de 5\%. A análise da composição das dietas da realimentação foi realizada pelo programa NUTRI versão 2,5, considerando-se as recomendações energético-protéicas da literatura para realimentação após jejum prolongado 12,20,21.

\section{RESULTADOS}

A idade dos 7 pacientes variou de 34 a 47 (média - $\mathrm{M}=41,6$ anos, desvio-padrão - $\mathrm{DP}=5,3$ ). No hospital, os pacientes mantiveram a recusa de alimentos, consumiram água ad libitum com adição $\mathrm{NaCl}$ (em média 8g/dia) a partir do $12^{\circ}$ dia do início do jejum, e $\mathrm{KCl}(27 \mathrm{mEq} / \mathrm{dia})$ a partir 
do $18^{\circ}$ do dia até o final do jejum; receberam também, por via oral, vitaminas $(C, A, D, E, K$, complexo $B$ ), ácido fólico, sulfato de magnésio, e fosfato monopotássico. No $41^{\circ}$ dia de jejum, a equipe multiprofissional iniciou administração endovenosa periférica de glicose a 5\% (1-2L/dia) + lípides a 10\% (415 a 830kcal/dia), conduta esta interrompida no $2^{\circ}$ dia de administração, devido à ocorrência de flebite nos membros superiores dos pacientes, relacionada à cateterização venosa. No $44^{\circ}$ dia do jejum, este foi interrompido pelos pacientes, que passaram a aceitar a realimentação por via oral.

Não se observou alteração significativa das variáveis antropométricas analisadas entre o início e o final da realimentação (Tabela 1). Para avaliar a adequação dos dados das medidas antropométricas, os resultados foram comparados com valores de referência para o sexo masculino: PCT $12,5 \mathrm{~mm}$ e para CB 29,3cm, IMC 18,5 a $24,9 \mathrm{~kg} / \mathrm{m}^{217,22}$.

Embora os valores da massa magra tenham apresentado uma queda de 4,4\% entre o $32^{\circ}$ dia do jejum e o $8^{\circ}$ dia da realimentação (Tabela 1), tal diferença não foi estatisticamente significante. Entretanto, observou-se variação significativa dos demais parâmetros da composição corpórea neste mesmo período.

Os resultados foram comparados com valores de referência para massa magra (80\%-86\%), gordura (14\%-20\%), água corpórea (55\%-65\%), valores de resistência (500 omhs) e reactância (50 omhs) $)^{18,19}$.

Os resultados de exames laboratoriais: glicemia, hemograma, proteínas totais e frações e eletrólitos coletados no início do jejum referemse ao $12^{\circ}$ dia (início), ou seja, no momento da internação dos pacientes, e os do final, os dados obtidos mais próximos do $43^{\circ}$ dia de jejum. Os dados referentes à realimentação foram os mais próximos do final da $1^{\mathrm{a}} \mathrm{e}$ da $2^{\mathrm{a}}$ semanas (Tabela 2).

Tanto a hemoglobina (HB) quanto o Hematócrito $(\mathrm{HT})$ apresentavam-se reduzidos no final do jejum em relação ao início, e ambos não se recuperaram durante a realimentação.

A CTL já se encontrava reduzida no início do jejum, e apresentou aumento significativo na realimentação, aproximando-se dos valores normais $\left(2.000 \mathrm{mil} / \mathrm{mm}^{3}\right)^{7}$.

A proteína total não se alterou, no entanto, a albumina sérica apresentava-se reduzida no final

Tabela 1. Medidas antropométricas e dados obtidos por boimpedância de pacientes no início e final do período de jejum prolongado e ao final da primeira e segunda semana de realimentação. São Paulo (SP), 2008.

\begin{tabular}{|c|c|c|c|c|c|c|c|c|c|}
\hline \multirow{3}{*}{ Variáveis } & \multicolumn{4}{|c|}{ Jejum } & \multicolumn{4}{|c|}{ Realimentação final } & \multirow{3}{*}{ Valor normal } \\
\hline & \multicolumn{2}{|c|}{ Início } & \multicolumn{2}{|c|}{ Fim } & \multicolumn{2}{|c|}{ Primeira semana \# } & \multicolumn{2}{|c|}{ Segunda semana \#\# } & \\
\hline & $M$ & $\mathrm{DP}$ & $M$ & $\mathrm{DP}$ & M & DP & M & DP & \\
\hline Peso $(\mathrm{kg})$ & 82,7 & 7,6 & 67,9 & $6,7 *$ & 70,3 & 5,9 & 73,2 & 6,6 & 78,6 \\
\hline $\mathrm{IMC}\left(\mathrm{kg} / \mathrm{m}^{2}\right)$ & 26,2 & 3,1 & 21,5 & $2,8^{*}$ & 22,6 & 2,8 & 23,2 & 2,7 & $18,5-24,9$ \\
\hline РCT $(\mathrm{mm})$ & 17,4 & 3,2 & 9,1 & $3,2^{*}$ & 10,9 & 4,2 & 12,4 & 3,9 & 12,5 \\
\hline $\mathrm{CB}(\mathrm{cm})$ & 30,0 & 1,4 & 28,9 & 2,1 & 28,0 & 2,5 & 28,7 & 1,7 & 29,3 \\
\hline Massa magra (\%) & - & & 72,9 & 1,7 & 68,5 & 9,9 & - & & $80,0-86,0$ \\
\hline Gordura (\%) & - & & 25,7 & 6,3 & 14,2 & $1,8^{*}$ & - & & $14,0-20,0$ \\
\hline Água corpórea total (\%) & - & & 53,6 & 4,3 & 62,4 & $1,9^{*}$ & - & & $55,0-65,0$ \\
\hline Resistência (omhs) & - & & 631,1 & 78,9 & 464,9 & $50,7^{*}$ & - & & 500,0 \\
\hline Reactância (omhs) & - & & 64,5 & 11,3 & 36,3 & $7,0^{*}$ & - & & 50,0 \\
\hline
\end{tabular}

M: média; DP: desvio-padrão; IMC: Índice de massa corporal; PCT: prega cutânea do tríceps; CB: circunferência do braço.

\#: $7^{\circ}$ dia; \# \#: $14^{\circ}$ dia da realimentação; * $p<0,05 ;$ ANOVA, teste de Bonferroni comparando o início e final do jejum. Os valores do peso e do IMC (início) referem-se ao $1^{\circ}$ dia do jejum, e as medidas da PCT (início) e CB (início) referem-se ao $12^{\circ}$ dia do jejum, ou seja, $1^{\circ}$ dia após a internação hospitalar; n:7; Bioimpedância (massa magra, gordura, água corporal, resistência e reactância) realizada no $32^{\circ}$ dia de jejum e $8^{\circ}$ dia de realimentação. 
Tabela 2. Parâmetros hematológicos de pacientes em jejum prolongado por 43 dias e ao final da primeira e segunda semana de realimentação. São Paulo (SP), 2008.

\begin{tabular}{|c|c|c|c|c|c|c|c|c|c|}
\hline \multirow{3}{*}{ Dosagens } & \multicolumn{4}{|c|}{ Jejum } & \multicolumn{4}{|c|}{ Realimentação } & \multirow{3}{*}{ Valor normal } \\
\hline & \multicolumn{2}{|c|}{ Inicial (12 dia) } & \multicolumn{2}{|c|}{ Final $\left(43^{\circ} \mathrm{dia}\right)$} & \multicolumn{2}{|c|}{ Final $1^{\mathrm{a}}$ semana } & \multicolumn{2}{|c|}{ Final $2^{\mathrm{a}}$ semana } & \\
\hline & M & DP & M & DP & M & DP & M & DP & \\
\hline Hemoglobina $(\mathrm{g} / \mathrm{dL})$ & 16,0 & 1,0 & 12,7 & $0,5^{*}$ & 10,8 & 0,34 & 11,6 & $0,7^{* *}$ & $13,0-18,0$ \\
\hline Hematócrito (\%) & 46,6 & 2,8 & 37,3 & $1,3^{*}$ & 31,8 & 1,50 & 34,9 & $2,0^{* *}$ & $40-52,0$ \\
\hline $\mathrm{CTL}\left(\mathrm{mil} / \mathrm{mm}^{3}\right)$ & 1301,0 & 413,4 & 954,1 & $241,5^{*}$ & 1732,0 & 253,00 & 1619,7 & $231,8^{* *}$ & $>2.000,0$ \\
\hline Proteína total (g/dL) & 5,9 & 0,4 & 5,7 & $0,3^{*}$ & 5,3 & 0,30 & nd & nd & $6,0-8,0$ \\
\hline Albumina ( $\mathrm{g} / \mathrm{dL}$ ) & 4,3 & 0,3 & 4,2 & $0,3^{*}$ & 3,3 & $0,30^{* *}$ & nd & nd & $3,5-5,0$ \\
\hline Fósforo (mEq/L) & 3,1 & 0,3 & 4,2 & $0,7^{*}$ & 2,4 & $0,60^{* *}$ & 3,9 & $0,6^{* * *}$ & $2,3-4,6$ \\
\hline Glicose $(\mathrm{g} / \mathrm{dL})$ & 65,7 & 7,6 & 101,1 & $10,2^{*}$ & 88,5 & 14,50 & 78,8 & $15,0^{* *}$ & $70,0-110,0$ \\
\hline
\end{tabular}

M: média; DP: desvio-padrão; CTL: contagem total de linfócitos; nd= não dosado.

${ }^{*} p<0,05$ (comparando $12^{\circ}$ e $43^{\circ}$ dia de jejum); ANOVA, Teste Tukey; $n=7 ;{ }^{* *} p<0,05$ (comparando $43^{\circ}$ dia de jejum e final da segunda semana de realimentação); ${ }^{* *} p<0,05$ (comparando final $1^{\text {a }}$ semana e final $2^{\text {a }}$ semana).

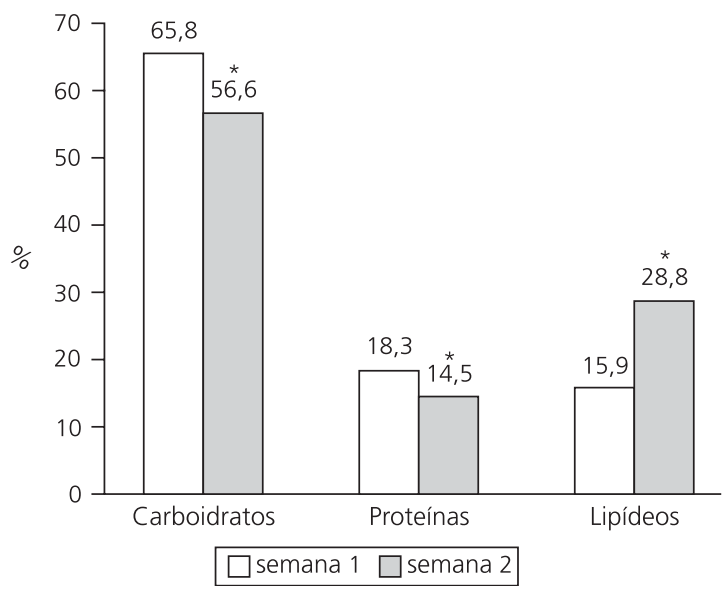

Figura 1. Composição de carboidratos, proteína e lipídeos das dietas utilizadas na realimentação de pacientes após jejum prolongado por 43 dias. São Paulo (SP), 2008.

Nota:Ingestão de hidratos de carbono, proteínas e lipídeos, expressa percentual do valor energético total. ${ }^{*} p<0,05$, ANOVA, teste Tukey comparando os valores da primeira semana com os da segunda semana de realimentação. $n=7$.

da primeira semana de realimentação. O fósforo sérico aumentou durante o jejum, reduziu-se na $1^{\circ}$ semana da realimentação, normalizando-se até o final da segunda semana (Figura 1).

Da primeira para a segunda semana, observou-se alteração significante $(p<0,05)$ no percentual de carboidratos, proteínas e lipídeos em relação ao valor energético total das dietas administradas (Figura 2). A ingestão energética foi calculada com base na composição química das dietas administradas diariamente.
As manifestações clínicas referem-se ao período de realimentação, e foram agrupadas em geral (ansiedade/depressão, edema de membros, fraqueza, hipotensão postural, flebite, sangramento gengival, herpes, cefaléia), e gastrointestinais (diarréia [3 ou mais evacuações líquidas/ dia], dor abdominal, flatulência, distensão, náuseas, vômito). Observou-se significativo aumento das manifestações gastrointestinais da primeira para a segunda semana, quando se analisou a totalidade de manifestações apresentadas diariamente em cada semana de realimentação observadas individualmente nos 7 pacientes estudados (Figura 3).

D I S C U S S Ã O

Jejum prolongado com uso de água e eletrólitos por até mais de 200 dias foi utilizado em meados do século XX para tratamento de obesidade ${ }^{8}$. Em indivíduos hígidos, não obesos, a sobrevida ao jejum por mais de 60 dias é escassa, sendo ademais limitado o detalhamento relatado das alterações fisiológicas e dos esquemas de realimentação utilizados ${ }^{4,8,23}$. O jejum prolongado, dependendo da sua duração, das condições nutricionais prévias dos pacientes, e da presença de doenças concomitantes, pode acarretar significativas alterações na composição corpórea. 


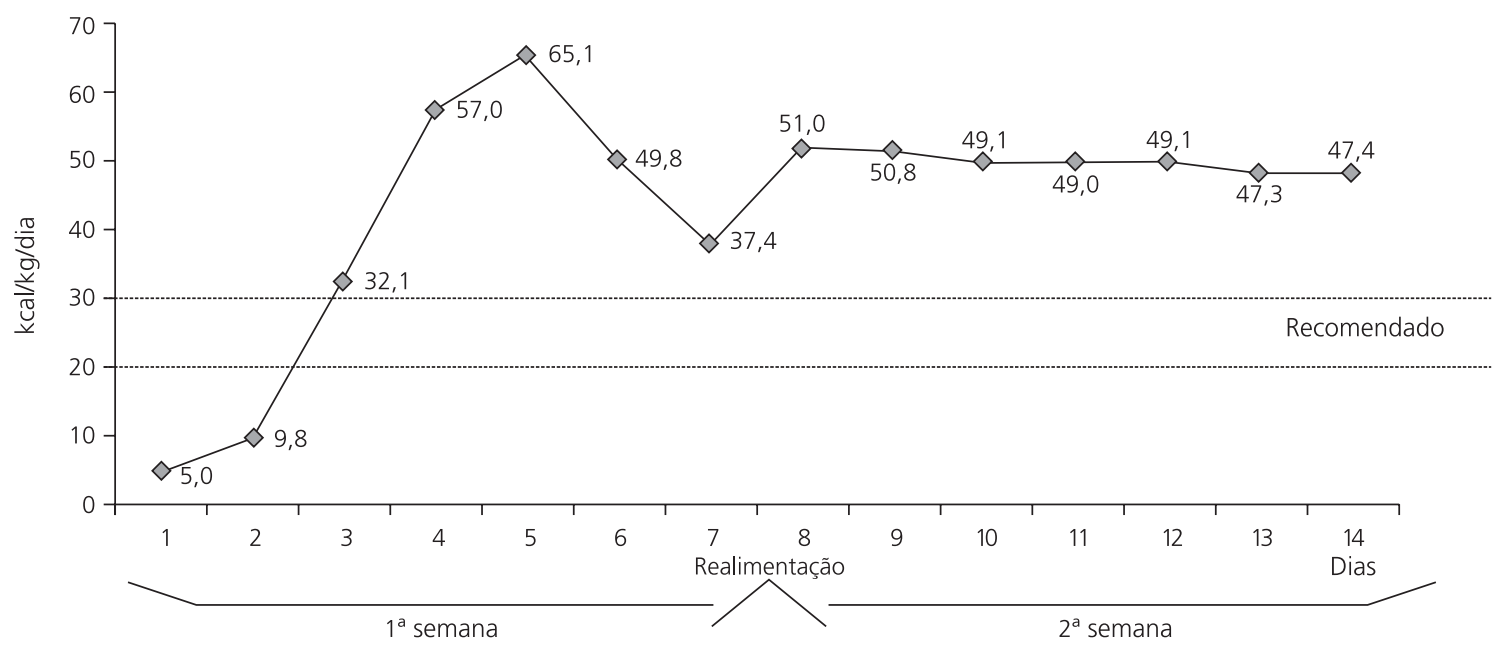

Figura 2. Ingestão energética diária durante a realimentação de pacientes após jejum prolongado por 43 dias. São Paulo (SP), 2008. Nota:kcal/kg de peso corpóreo dos pacientes/dia; N: 7; A média da ingestão energética da primeira semana foi maior que a da segunda semana. $p<0,05$, ANOVA, Método Dunns.

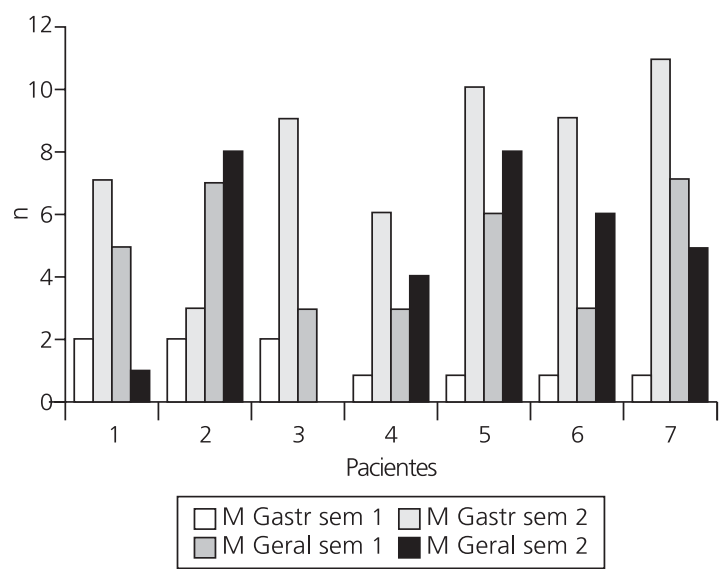

Figura 3. Manifestações gerais e gastrintestinais apresentadas pelos pacientes em duas semanas de realimentação após jejum prolongado por 43 dias. São Paulo (SP), 2008.

Nota:M: manifestação; Gast: gastrintestinal; sem: semana. Manifestações gerais e gastrintestinais observadas por paciente $(n=7)$, expressas em número de episódios na primeira e na segunda semanas da realimentação, e representadas pela média e desvio-padrão. Observou-se que o total de manifestações gastrintestinais dos 7 pacientes, ocorridas na segunda semana, foi significativamente maior que o total da primeira semana; nas manifestações gerais, tal aumento não foi significativo (ANOVA, Teste de Bonferroni, $p<0.05$ ).

São relatadas perdas de peso entre $20 \%$ $25 \%$ em períodos de 40 a 60 dias de jejum, portanto semelhantes ao do presente estudo em que, ao iniciar o jejum, os pacientes encontravam- se com sobrepeso (IMC $\left.26,2 \mathrm{~kg} / \mathrm{m}^{2} \mathrm{DP}=3,1\right)$ e, embora com significativa redução de peso $(18,0$ $\mathrm{DP}=2,3 \%$ ) e de tecido adiposo (redução da PCT

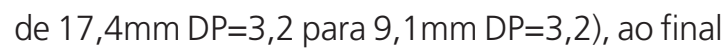
do jejum encontravam-se em eutrofia (IMC $22,6 \mathrm{~kg} / \mathrm{m}^{2} \mathrm{DP}=2,8$ ) (Tabela 1). Observou-se, entretanto, preservação da massa muscular representada pela medida da circunferência do braço.

A avaliação pela bioimpedância (BIA) realizada no $32^{\circ}$ dia do jejum e no $8^{\circ}$ dia da realimentação mostrou significante redução $(p<0,05)$, da gordura $(p<0,05)$. Embora a média dos valores da massa magra tenha apresentado redução de 4,4\% nesse período, tal alteração não foi estatisticamente significante, e não foram registrados indícios de repercussão clínica da redução observada (Tabela 1). Apesar de a presença de edema nos pacientes poder comprometer os resultados da avaliação destes compartimentos corpóreos (massa magra e gordura), os resultados foram compatíveis com a avaliação antropométrica realizada nos pacientes. Ademais, a BIA realizada no $32^{\circ}$ dia do jejum, mostrou que os pacientes apresentavam valores de água corporal abaixo dos valores considerados normais. Já no $8^{\circ}$ dia da realimentação, a água corporal estava aumentada 
em relação ao $32^{\circ}$ dia do jejum, fato coerente com o rápido aumento do peso corpóreo e de edema observados entre o final do jejum e o $7^{\circ}$ dia da realimentação, além da redução observada no valor de resistência observado (Tabela 1).

A utilização de fórmula com glutamina na realimentação tem sido indicada, pois admite-se que, comparada com dieta sem adição deste aminoácido, ela promova melhor recuperação da mucosa intestinal, geralmente alterada pelo jejum prolongado, e também aumento da contagem de linfócitos, estimulando assim a função imune ${ }^{24,25}$. No presente estudo, dieta da primeira semana, contendo $0,5 \mathrm{~g}$ de glutamina por $\mathrm{kg}$ de peso corpóreo ao dia, conforme recomendação da literatura 26,27 , foi bem aceita e tolerada pelos pacientes. Neste período, os pacientes apresentaram significativo aumento na contagem total de linfócitos e número reduzido de manifestações gastrointestinais relatadas (Tabela 2, Figura 3).

Sabe-se que, no jejum prolongado ocorre redução da produção das enzimas digestivas, da digestão de dissacarídeos e gorduras e da absorção de nutrientes, bem como redução do transporte de aminoácidos através da mucosa intestinal, associada ao aumento da permeabilidade desta ${ }^{9,24}$. Estas alterações interferem no processo inicial de realimentação, fato que justifica cuidados com a evolução das dietas, sobretudo quanto à reintrodução de alimentos fontes de lactose, sacarose, e de alimentos gordurosos ${ }^{13}$.

Sintomas associados a intolerâncias alimentares incluem, freqüentemente, flatulência, diarréia, cólicas e distúrbios gastrointestinais ${ }^{10,28}$, os quais foram relatados pelos pacientes em maior proporção, com aumento siginificativo na segunda semana de realimentação, observados diariamente/paciente e analisados na totalidade (Figura 3).

No presente estudo, as dietas da segunda semana apresentam conteúdo lipídico significativamente maior que $\mathrm{o}$ da $1^{\circ}$ semana (Figura 1). Apesar de considerado normal para pessoas saudáveis, possivelmente foi excessivamente ele- vado após jejum prolongado, em função da recuperação, provavelmente, inadequada da disfunção digestiva9 .

Embora a aceitação das dietas tenha sido registrada nos prontuários como boa, adequada ao apetite dos pacientes, cabe, porém, observar que estudos clínicos e experimentais relatam a ocorrência de hiperfagia na realimentação após jejum prolongado, sendo ela considerada um mecanismo de restauração da perda de tecido adiposo decorrente do jejum, e que regride com a recuperação da gordura e da massa magra ${ }^{29,30}$. No presente estudo, não se observou recuperação significativa de peso nem de tecido adiposo em relação às perdas decorrentes do jejum (Tabela 1), apesar do aumento de apetite relatado pelos pacientes na primeira semana de realimentação e do elevado aporte energético das dietas administradas (Figura 2). Nas duas primeiras semanas de realimentação os pacientes apresentaram um ganho de, aproximadamente, $5 \mathrm{~kg}$ de peso corpóreo (Tabela 1), e presença de edema, fato que pode ser atribuído à retenção hídrica e ao excesso de calorias ingeridas, em relação às recomendações ${ }^{13}$. A média de ingestão energética diária da primeira semana foi significativamente maior que a da segunda, $49 \mathrm{kcal} / \mathrm{kg} / \mathrm{dia}$ DP=15 e $37 \mathrm{kcal} /$ $\mathrm{kg} /$ dia DP=23, respectivamente (Figura 2).

Assim, na fase inicial da realimentação após jejum prolongado, parece justificável controlar o aporte energético administrado, mesmo com aumento do apetite e boa aceitação da dieta, visando a evitar aporte excessivo de calorias, rápido ganho de peso às custas de retenção hídrica e risco da síndrome da realimentação.

Observou-se, neste estudo, que os níveis séricos de fósforo estavam significativamente reduzidos no $4^{\circ}$ dia da realimentação, no período em que os pacientes estavam recebendo aporte energético mais elevado ${ }^{13,14}$ tendo-se observado normalização dos níveis séricos do fósforo na segunda semana, coincidente com a redução do aporte energético ofertado (Tabela 2, Figura 2). 
Apesar da queda dos níveis séricos do fósforo, não se verificou a síndrome da realimentação. Embora o conteúdo energético administrado aos pacientes deste estudo tenha se situado acima do recomendado para a realimentação após jejum prolongado ${ }^{13,14}$, a dieta da $1^{\circ}$ semana teve boa aceitação e tolerância, e permitiu o aumento significativo da contagem total de linfócitos (Tabela 2, Figuras 2 e 3). A glicose sérica apresentou aumento durante o jejum (Tabela 2), achado não usual em situação de ausência de ingestão, e que pode ser atribuído à administração de nutrição parenteral periférica nos 2 últimos dias do jejum, normalizando em seguida com a suspensão da mesma e introdução de dieta via oral.

\section{O N CLUS Ã O}

O esquema de realimentação utilizado promoveu resultado global adequado, apesar do reduzido número de pacientes estudados. Mais estudos clínicos são necessários para reajuste no aporte energético administrado, e melhor definição do momento para reintrodução de lactose e de dieta normolipídica.

\section{COLABORADORES}

N.D. PUCCI idealizou o trabalho e a elaboração de todas as etapas, o qual foi finalizado como tese de dissertação de mestrado. B. FONTES revisou e orientou todas as etapas do trabalho, desde o projeto até a redação final do mesmo. R.S. POGGETTI participou na análise dos dados e na orientação da redação, da discussão, dos resultados e da conclusão.

\section{REFER Ê N CIAS}

1. Levenson SM, Seifter E. Inanição: respostas metabólicas e fisiológicas. In: Fischer JE. Nutrição em cirurgia. São Paulo: Medsi; 1985. p.369-416.

2. Gavranich KC, Petrucci PHG, Pucci ND, Maculevicius J. Avaliação do déficit calórico ocasionado pelo preparo dietético de exames. Anais do Congresso Brasileiro de Nutrição Enteral e Parenteral; 1993. Canela, RS.
3. Waitzberg DL, Caiaffa WT, Correia MITD. Hospital malnutrition: the Brazilian national survey (IBRANUTRI): a study of 4000 patients. Nutr. 2001; 17(7):573-80.

4. Frommel DL, Questiaux E, Gautier M, Schwarzenberg L. Voluntary total fasting: a challenge for the medical community. Lancet. 1984; 1(8392): 1451-2

5. Schnitzker MA, Mattman PE, Bliss TLA. A clinical study of malnutrition in Japanese prisioners of war. Ann Int Med. 1951; 35(1):69-96.

6. Pucci ND, Mazzoneto EC, Maculevicius J. Alterações metabólicas e nutricionais no jejum prolongado voluntário. Rev Bras Nutr Clin. 1999; 14(1):33.

7. Moreira JC, Waitzberg DL. Conseqüências funcionais da desnutrição. In: Waitzberg DL. Nutrição oral, enteral e parenteral na prática clínica. 3a. ed. São Paulo: Atheneu; 2001.

8. Thonson TJ, Runcie J, Miller V. Treatment of obesity by total fasting for up to 249 days. Lancet. 1966; 2(7471):992-6.

9. Ahlman B, Andersson K, Leijonmarck C, Ljungqvist O, Hedenborg I, Wernerman J. Short-term starvation alters the free amino acid content of human intestinal mucosa. Clin Sci. 1994; 86(6): 653-62.

10. Evangelista J. Emprego incorreto dos alimentos. In: Evangelista J. Alimentos, um estudo abrangente. São Paulo: Atheneu; 1992.

11. Brozek J, Chapman CB, Keys, AD. Drastic food restrition. JAMA. 1948; 137(18):1569-74.

12. Kirby DF. Physician Workshop, Refeeding Syndrome: background, diagnoses and manegement. Proceeding of the $19^{\text {th }}$ Clinical Congress: 1995; Miami, Florida, p.220-5.

13. Apovian CM, MC Mahon MM, Bristrian BR. Guidelines for refeeding the marasmatic patient. Crit Care Med. 1990; 18(9):1030 -3.

14. Weinsier RL, Krundieck CL. Death resulting from overzealous total parenteral nutrition: refeeding syndrome revisted. Am J Clin Nutr. 1981; 34(3): 393-9.

15. São Paulo. Governo do Estado de São Paulo Hospital das Clínicas. In: Manual de dietas do complexo HC. São Paulo: Governo do Estado de São Paulo; 1980. p.1-122.

16. Jellife $D$. The assessment of the nutritional status of the community. Geneve: WHO; 1966.

17. World Health Organization: Obesity, preventing and managing the global epidemic. Geneve: WHO; 1998. 
18. Tbw-Newmed. Byodinamics model 310. Manual de instrução do monitor de composição corpórea. São Paulo; 1994.

19. Mattar JA. Bioimpedância, reatância e resistência: parâmetros biofísicos úteis em suporte nutricional e medicina intensiva. Rev Metab Nutr. 1995; (2): 58-62.

20. Sigma Stat [computer program]. Version 2,03: Statistical software. SPSS-INC. 1992-97.

21. Universidade Federal de São Paulo. Escola Paulista de Medicina. Nutri [Programa de computador]. Versão 2,5: programa de apoio à decisão em nutrição para cálculo de dietas. São Paulo; 1995.

22. Blackburn GI, Bistrian BR. Nutritional and metabolic assessment of the hospitalized patient. JPEN J Parenter Enteral Nutr. 1977; 1(1):11-32.

23. Peel M. Hunger strikes. BMJ. 1997; 315(7112): 829-30.

24. Buchman Al, Moukarzel AA, Bhuta S, Belle M, Ament ME, Eckhert CD, et al. Parenteral nutrition is associated with intestinal morphologic and functional changes in humans. JPEN J Parenter Enteral Nutr. 1995; 19(6):453-60.

25. Sarac TP, Souba WW, Miller J, Ryan CK, Koch M, Bessey $P Q$, et al. Starvation induces differential small bowel luminal amino acid transport. Surgery. 1994; 116(4):679-86.

26. Savy G. Enteral glutamine supplemention; clinical review and practical guidelines. Nutr Clin Prac. 1997; 12(6):259-62.

27. Daurea AD, Lewis JG. Intestinal premeability and systemic infecctions in critically ill patients:effect of glutamine. Crit Care Med. 2005; 33(5): 1125-35.

28. Kerndt PR, Naughton JL, Dirscoll, CE, Loxterkamp DA.Fasting: history, pathophysiology and complications medical progress. West J Med. 1982; 137(5):379-99.

29. Duloo AG, Jacquet J, Girardier L. Postarvation hyperfagia and body fat overshooting in humans: a role for feedback signals from lean and fat tissues. Am J Clin Nut. 1997; 65(3):717-23.

30. Per Bjorntorp, Yang M. Refeeding after fasting in rat: effects on body composition and food efficiency. Am J Clin Nutr. 1982; 36(3):444-9.

Recebido em: 24/8/2006

Versão final reapresentada em: 14/12/2007 Aprovado em: 23/7/2008 